\title{
Non-leptonic charmless $B_{c}$ decays (and their search at $\mathrm{LHCb}$ )
}

\section{S. Descotes-Genon*}

Laboratoire de Physique Théorique, CNRS/Univ. Paris-Sud 11 (UMR 8627),

91405 Orsay, France

E-mail: descoteseth.u-psud.fr

\section{J. He, E. Kou and P. Robbe}

Laboratoire de l'Accélérateur Linéaire, Université Paris-Sud 11, CNRS/IN2P3 (UMR 8607),

91405 Orsay, France

We discuss the decay of $B_{c}$ mesons into two light mesons $\left(\pi, K^{(*)}, \eta^{(\prime)}, \rho, \omega, \phi\right)$. All these decay channels come from a single type of diagram, namely tree annihilation. This allows us to derive extremely simple $S U(3)$ relations among these processes. The size of annihilation contributions is an important issue in $B$ physics, and we provide two different estimates in the case of non-leptonic charmless $B_{c}$ decays, either a comparison with annihilation decays of heavy-light mesons or a perturbative model inspired by QCD factorisation. We finally discuss a possible search for these channels at LHCb.

The 2009 Europhysics Conference on High Energy Physics,

July 16 - 222009

Krakow, Poland

${ }^{*}$ Speaker. 
Since the first observation of the $B_{c}$ meson in 1998 from the CDF collaboration, its mass and width have been measured, and bounds on some non-leptonic channels have been set $(J / \psi$ with one or three pions, $D^{*+} \bar{D}^{0} \ldots$ ). The $B_{c}$ meson shares many features with the better studied quarkonia, with the significant difference that its decays are not mediated through strong interaction but weak interaction due to its flavour quantum numbers $B=-C= \pm 1$. The properties of the $B_{c}$ meson will be further scrutinized by the LHC experiments; the high luminosity of the LHC machine opens the possibility to observe many $B_{c}$ decay channels, in particular at $\mathrm{LHCb}$. We report here on recent investigations on the charmless non-leptonic $B_{c}$ decays [1].

The diagram for the non-leptonic charmless $B_{c}$ decays is shown in fig. 1 . The initial $\bar{b}$ and $c$ quarks annihilate into $u$ and $\bar{d}$ or $\bar{s}$ quarks, which form two light mesons by hadronising with a pair of $q \bar{q}(q=u, d, s)$ emitted from a gluon. There are 32 decay channels of this kind if we consider only the lightest pseudoscalar and vector mesons. In the case of two outgoing pseudoscalar mesons (PP), there are 4 modes with strangeness one: $K^{+} \pi^{0}, K^{+} \eta, K^{+} \eta^{\prime}, K^{0} \pi^{+}$and 4 modes with strangeness zero: $\pi^{+} \pi^{0}, \pi^{+} \eta, \pi^{+} \eta^{\prime}, K^{+} \bar{K}^{0}$. The same applies for two vectors (VV) up to the obvious changes: $K^{*+} \rho^{0}, K^{*+} \phi, K^{*+} \omega, K^{* 0} \rho^{+}, \rho^{+} \rho^{0}, \rho^{+} \phi, \rho^{+} \omega, K^{*+} \bar{K}^{* 0}$. In the case of VP decays, one can get 8 strange decay modes $\left(\Delta S=1\right.$ processes): $K^{*+} \pi^{0}, K^{*+} \eta, K^{*+} \eta^{\prime}, K^{* 0} \pi^{+}, \rho^{0} K^{+}, \phi K^{+}, \omega K^{+}, \rho^{+} K^{0}$ and 8 non-strange decay modes ( $\Delta S=0$ processes): $\rho^{+} \pi^{0}, \rho^{+} \eta, \rho^{+} \eta^{\prime}, K^{*+} \bar{K}^{0}, \rho^{0} \pi^{+}, \phi \pi^{+}, \omega \pi^{+}$, $\bar{K}^{* 0} K^{+}$. These 32 non-leptonic charmless $B_{c}$ decays are all pure annihilation and come from a single-tree annihilation diagram. Understanding these channels can have an important impact on the discussion of annihilation in other decays, in particular for heavy-light mesons, where similar contributions come from several operators and interfere with other mechanisms.

Compared to the case of $B_{u, d}$ decays, the $S U(3)$ relations for the $B_{c}$ decays are extremely simple and can be derived [1] from the Wigner-Eckart theorem following ref. [2]. Taking into account Clebsch-Gordan coefficients and octet-singlet mixing, we get the $V P$ amplitudes:

$\begin{array}{cccc}\text { Mode } & \text { Amplitude } & \text { Mode } & \text { Amplitude } \\ K^{+*} \pi^{0} & \frac{1}{2} \sqrt{\frac{3}{5}} S^{V P}+\frac{1}{2 \sqrt{3}} A^{V P} & \omega K^{+} & -\frac{1}{2 \sqrt{15}} S^{V P}-\frac{1}{2 \sqrt{3}} A^{V P}+\sqrt{\frac{2}{3}} I^{V L} \\ \rho^{0} K^{+} & \frac{1}{2} \sqrt{\frac{3}{5}} S^{V P}-\frac{1}{2 \sqrt{3}} A^{V P} & \phi K^{+} & \frac{1}{\sqrt{30}} S^{V P}+\frac{1}{\sqrt{6}} A^{V P}+\frac{1}{\sqrt{3}} I^{V P} \\ K^{*+} \eta & -\frac{1}{3} \sqrt{\frac{2}{5}} S^{V P}+\frac{\sqrt{2}}{3} A^{V P}+\frac{1}{3} I^{V P} & \rho^{+} K^{0} & \sqrt{\frac{3}{10}} S^{V P}-\frac{1}{\sqrt{6}} A^{V P} \\ K^{*+} \eta^{\prime} & \frac{1}{6 \sqrt{5}} S^{V P}-\frac{1}{6} A^{V P}+\frac{2 \sqrt{2}}{3} I^{V P} & K^{* 0} \pi^{+} & \sqrt{\frac{3}{10}} S^{V P}+\frac{1}{\sqrt{6}} A^{V P} \\ \text { Mode } & \text { Amplitude } & \text { Mode } & \text { Amplitude } \\ \rho^{+} \pi^{0} & \frac{1}{\sqrt{3}} A^{V P} & \omega \pi^{+} & \frac{1}{\sqrt{15}} S^{V P}+\sqrt{\frac{2}{3}} I^{V P} \\ \rho^{0} \pi^{+} & -\frac{1}{\sqrt{3}} A^{V P} & \phi \pi^{+} & -\sqrt{\frac{2}{15}} S^{V P}+\frac{1}{\sqrt{3}} I^{V P} \\ \rho^{+} \eta & \frac{2}{3} \sqrt{\frac{2}{5}} S^{V P}+\frac{1}{3} I^{V P} & K^{*+} \bar{K}^{0} & \sqrt{\frac{3}{10}} S^{V P}-\frac{1}{\sqrt{6}} A^{V P} \\ \rho^{+} \eta^{\prime} & -\frac{1}{3 \sqrt{5}} S^{V P}+\frac{2 \sqrt{2}}{3} I^{V P} & \bar{K}^{* 0} K^{+} & \sqrt{\frac{3}{10}} S^{V P}+\frac{1}{\sqrt{6}} A^{V P}\end{array}$

The amplitudes are still to be multiplied by $G_{F} / \sqrt{2}$ and a CKM factor $V_{u D} V_{c b}^{*}(D=d$ or $s)$. We get the relations

$$
\begin{aligned}
A\left(B_{c}^{+} \rightarrow K^{* 0} \pi^{+}\right) & =\sqrt{2} A\left(B_{c}^{+} \rightarrow K^{*+} \pi^{0}\right)=\hat{\lambda} A\left(B_{c}^{+} \rightarrow \bar{K}^{* 0} K^{+}\right) \\
A\left(B_{c}^{+} \rightarrow \rho^{+} K^{0}\right) & =\sqrt{2} A\left(B_{c}^{+} \rightarrow \rho^{0} K^{+}\right)=\hat{\lambda} A\left(B_{c}^{+} \rightarrow K^{*+} \bar{K}^{0}\right)
\end{aligned}
$$




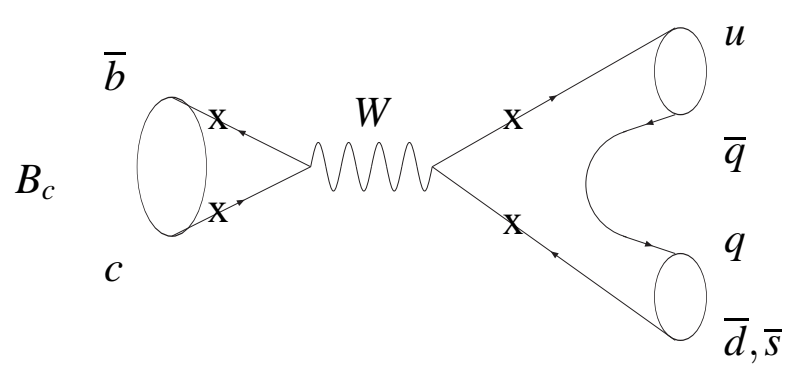

Figure 1: Generic diagram for the non-leptonic charmless $B_{c}$ decays

Due to the Wigner-Eckhart theorem and the involvement of a single operator, we get all the $P V$ amplitudes in terms of three different reduced matrix elements $S^{V P}, A^{V P}$ and $I^{V P}$. One can simplify further the expressions by assuming the validity of the Zweig rule for the $\Delta S=0$ processes involving $\phi$, e.g., setting $\operatorname{Br}\left(B_{c}^{+} \rightarrow \phi \pi^{+}\right)=0$ to obtain a further relation between $I^{V P}$ and $S^{V P}$. Similar relations can be derived in the case of $P P$ modes and $V V$ modes, involving different reduced matrix elements for $P P$ and for the different partial waves (or helicity amplitudes) for $V V$. The $S U$ (3) relationships allow one to relate different modes belonging to the same multiplets, but not to fix the overall scale of the reduced matrix elements, and thus that of the branching ratios. We followed two different paths to estimate these matrix elements, by using experimental data on pure annihilation $B$ decays and by relying on a one-gluon picture à la QCD factorisation.

There are two pure annihilation processes observed in heavy-light $B$ decays: $B^{0} \rightarrow K^{+} K^{-}$and $B^{0} \rightarrow D_{s}^{-} K^{+}$. Since we are interested in charmless final states, we can try and compare $B_{c} \rightarrow K^{+} \bar{K}^{0}$ and $B^{0} \rightarrow K^{+} K^{-}$processes. After relying on further hypotheses described in ref. [1], we obtain the following branching ratios of interest:

$$
\begin{aligned}
{\left[B_{d} \text { annihil }\right] \quad } & B R\left(B_{c} \rightarrow \phi K^{+}\right) \simeq \mathscr{O}\left(10^{-7}-10^{-8}\right), \quad B R\left(B_{c} \rightarrow \bar{K}^{* 0} K^{+}\right) \simeq \mathscr{O}\left(10^{-6}\right) \\
& B R\left(B_{c} \rightarrow \bar{K}^{0} K^{+}\right) \simeq \mathscr{O}\left(10^{-6}\right), \quad B R\left(B_{c} \rightarrow \bar{K}^{* 0} K^{*+}\right) \simeq \mathscr{O}\left(10^{-6}\right)
\end{aligned}
$$

A second method consists in a model based on one-gluon exchanges, in close relation with the model proposed in QCD factorisation to estimate annihilation contributions for the decays of heavy-light mesons. As described in ref. [1], we found that: $i$ ) the charmless $B_{c}$ decays are much simpler, since the only operator contributing is $\mathrm{O}_{2}$, and there is only one combination of CKM factors ( $V_{c b}^{*} V_{u D}$ where $D=d, s$ depending on the strangeness of the outgoing state), and $\left.i i\right)$ the longdistance divergences, which prevent us from estimating the annihilation contribution accurately in $B_{u, d}$ decays, do not arise in $B_{c}$ annihilation (they are tamed by the nonvanishing charm quark mass). The expressions for all the decay channels were recovered from refs. [4] if we identify between the Wigner-Eckart reduced matrix elements $S, I, A$ and the $O_{2}$ reduced coefficients $b_{2}$. However, the uncertainty coming from endpoint divergences in the case of $B_{u, d, s}$ decays is absent here, so that the estimation of $B_{c}$ annihilation in this framework suffers from smaller uncertainties than for heavylight decays. We obtain finally the following values of branching ratios:

$$
\begin{aligned}
\text { [One-gluon] } & B R\left(B_{c} \rightarrow \phi K^{+}\right)=5 \times 10^{-9}, \quad B R\left(B_{c} \rightarrow \bar{K}^{* 0} K^{+}\right)=9.0 \times 10^{-8} \\
& B R\left(B_{c} \rightarrow \bar{K}^{0} K^{+}\right)=6.3 \times 10^{-8}, B R\left(B_{c} \rightarrow \bar{K}^{* 0} K^{*+}\right)=9.1 \times 10^{-8}
\end{aligned}
$$


Our estimates of the branching ratios in the above two ways are not consistent. This stems from the conceptual differences between two methods. The method based on $B_{d}$ annihilation treats the charm quark as massless. It takes into account some of the non-perturbative long-distance effects expected to occur in $B_{d}$ and $B_{c}$ decays, but treats the relation between matrix elements of the operators $O_{1}$ and $O_{2}$ in a very naïve way. It relies also on extremely naive assumptions concerning the respective size of matrix elements for $P P, V P$ and $V V$ modes. The method based on one-gluon exchange treats the charm quark as heavy. It assumes the dominance from a specific set of diagrams computed in a perturbative way within a consistent framework. It is well known that both kinds of estimation yield rather different results. This is illustrated by the fact that the estimate of $B_{d} \rightarrow$ $K^{+} K^{-}$in the annihilation models of QCD factorisation (around $10^{-8}$ with substantial uncertainties) is one order of magnitude below the current experimental average. There are well-known cases where final-state interaction can increase significantly estimates based on factorisation, for instance $B \rightarrow K \chi_{c}$ or $D_{s}^{+} \rightarrow \rho^{0} \pi^{+}$. Therefore, an observation of the non-leptonic charmless $B_{c}$ decays will certainly have an important key to clarify such a controversy.

We can estimate the expected sensitivity of LHCb for a specific channel. Taking into account the trigger and reconstruction efficiencies, as well as Zweig-rule and Cabibbo suppression, we draw the conclusion that the $B_{c}^{+} \rightarrow \bar{K}^{* 0} K^{+}$channel might be the best candidate for the detection. Since the selection criteria and trigger efficiencies are different for each channel, detailed simulations are necessary in order to estimate the expected sensitivity for different channels. For example, such a study has been done for $B_{c} \rightarrow J / \psi \pi^{+}$[5]. From the expected branching ratio $\operatorname{Br}\left(B_{c} \rightarrow\right.$ $\left.J / \psi \pi^{+}\right) \simeq 1 \%$, it was deduced that over a thousand of events are expected after the one year run of LHCb. By scaling this observation to the processes of interest, we can very roughly estimate that an assumption of $\operatorname{Br}\left(B_{c}^{+} \rightarrow \bar{K}^{* 0} K^{+}\right)=10^{-6}$ yields a few events per year at LHCb. The analysis of LHCb data will thusset first experimental limits on the non-leptonic charmless $B_{c}$ decays, and give hints on the annihilation mechanisms at work in these decays.

\section{Acknowledgments}

Work supported in part by EU Contracts No. MRTN-CT-2006-035482 ("FLAVIAnet") qnd MIF1-CT-2006-027144 and by the ANR contracts "DIAM" ANR-07-JCJC-0031 and "LFV-CPVLHC” ANR-NT09-508531.

\section{References}

[1] S. Descotes-Genon, J. He, E. Kou and P. Robbe, arXiv:0907.2256 [hep-ph].

[2] D. Zeppenfeld, Z. Phys. C 8, 77 (1981).

[3] J. J. de Swart, Rev. Mod. Phys. 35, 916 (1963) [Erratum-ibid. 37, 326 (1965)].

[4] M. Beneke and M. Neubert, Nucl. Phys. B 675, 333 (2003) [arXiv:hep-ph/0308039]. M. Beneke, J. Rohrer and D. Yang, Nucl. Phys. B 774, 64 (2007) [arXiv:hep-ph/0612290].

[5] O. P. Yushchenko, Note LHCb 2003-113.

Y. Gao, J. He, Z. Yang. Note LHCb-2008-077 ; CERN-LHCb-2008-077. 